\title{
Factores que Influyen en la Decisión de Ingreso a la Universidad
}

\section{Issues that affect the decision to enroll in higher education}

Presentación: 01/10/2020

Aprobación: 24/11/2020

\section{María Marta Coria}

Universidad Tecnológica Nacional - Argentina

mariamarta.coria@gmail.com

\section{Resumen}

El trabajo investiga los factores que influyen en la decisión de comenzar estudios de educación superior y analiza el caso de la Extensión Áulica San Martín de los Andes de la Facultad Regional del Neuquén de la Universidad Tecnológica Nacional. Se realizó una encuesta para recabar información sobre las características socioeconómicas de los estudiantes, los motivos para ingresar a la universidad y sus expectativas futuras. Los resultados muestran que las principales motivaciones para estudiar corresponden a factores instrumentales vinculados con el deseo y la necesidad de contar con un título de educación superior y a cuestiones psicosociales, en particular, el ideario de la educación superior como medio para lograr la movilidad social y conseguir mejores oportunidades laborales. También ponen de manifiesto la relevancia de contar con ofertas educativas locales en contextos donde prima una baja movilidad estudiantil por motivos económicos, familiares o idiosincráticos.

Palabras clave: educación superior, ingreso a la universidad, capital humano, estudio de casos

\begin{abstract}
This paper analyses factors that influence the decision of enrolling in higher education. It is described the case of the Neuquén Regional Faculty of the National Technological University located in San Martín de los Andes. . A survey was conducted to gather information about the socioeconomic characteristics of the students, the reasons for entering the university and their future expectations. The results obtained show that the main motivations for studying are: instrumental reasons linked to the desire and the need of having a higher education degree, as well as psychosocial issues, particularly regarding higher education as a way to achieve social mobility and better job opportunities. Local educational offers are also highlighted in places where due to economic, familiar or idiosyncratic reasons there is low student mobility.
\end{abstract}

Keywords: higher education, tuition, human capital, case study 


\section{INTRODUCCIÓN}

Uno de los fenómenos más significativos en el ámbito de la educación superior en los últimos años es su tendencia a la masificación. Entre las razones que explican este fenómeno pueden mencionarse un aumento de la riqueza a nivel mundial y más políticas estatales propicias, en particular aquellas que apuntan a mejorar la equidad social (IIEP - UNESCO, 2017). Asimismo, las mejores tasas de matriculación en la enseñanza primaria y la secundaria son un factor que explica el aumento de la demanda de educación superior dado que, a medida que más estudiantes completan los ciclos de la enseñanza secundaria, aumenta la cantidad de personas que están en condiciones de acceder a estudios superiores. Otro factor es el aumento de la participación en la enseñanza superior de estudiantes no tradicionales, es decir, estudiantes de tiempo parcial y adultos que trabajan; los adultos (personas de más de 25 años) constituyen una parte importante de los estudiantes universitarios en diversos países europeos.

El aumento de la cantidad de estudiantes de educación superior es también un fenómeno relevante en Argentina. En los últimos 30 años la cantidad de estudiantes creció exponencialmente y la tasa de crecimiento anual supera ampliamente la de crecimiento poblacional. La cantidad de estudiantes que cursa carreras de grado y pregrado creció un 29\% entre 2005 y 2017, alcanzando el máximo histórico de 2 millones en ese último año de acuerdo con las cifras del Ministerio de Educación de la Nación. El 79\% cursa estudios en instituciones de gestión estatal, que son, a su vez, la mayoría del sistema (66 sobre un total de 131) y el resto en instituciones de gestión privada. El 50\% de los estudiantes tienen menos de 25 años mientras que el 26\% tienen más de 30 años.

La cantidad de nuevos inscriptos, es decir, los que ingresan al sistema de educación superior, muestra una tendencia creciente en los últimos 30 años. Según datos del Ministerio de Educación de la Nación, en 2017 (último año disponible) se registra el número máximo de ingresantes a carreras de grado y pregrado, con 516.305 nuevos inscriptos, lo que representa un aumento del $43 \%$ respecto del año 2005. Siguiendo con los datos del año 2017, la mayoría son estudiantes menores a 25 años (66\%) y estudian en el subsistema de gestión pública $(76 \%)$.

El aumento de la población estudiantil se verifica en un contexto caracterizado por políticas institucionales orientadas a garantizar el derecho a la educación superior, tales como la obligatoriedad del nivel medio (Ley $\mathrm{N}^{\circ} 26.206$ del año 2006), la gratuidad de los estudios de grado y pregrado en las instituciones de gestión pública, y el acceso, a la mayoría de las instituciones, sin examen que condicionen el ingreso ni cupos de ingreso. En los últimos 25 años, otra política de ampliación de las oportunidades educativas fue la regionalización de la oferta tras la creación de universidades y sedes en diferentes regiones del país (García de Fanelli, 2015) y la diversificación de la oferta, con la creación de nuevas carreras que ofrecen más opciones a los estudiantes y permiten orientar mejor la elección vocacional.

En Argentina, la escolaridad obligatoria abarca 13 años, desde los 5 años de edad hasta la finalización de la educación secundaria. La educación superior no es obligatoria y por lo tanto su demanda depende de cada persona.

La teoría del capital humano explica la demanda de educación como resultado de los retornos que ésta otorga, en particular, en los niveles de ingreso futuros. Según esta teoría, la educación mejora las capacidades y habilidades del individuo y esto le permite conseguir empleos mejor remunerados, estableciéndose así una relación causal y positiva entre el nivel educativo y los ingresos del individuo (Morduchowicz, 2004). Desde esta perspectiva, la 
demanda de educación superior depende de las expectativas sobre el mercado laboral, en particular, de obtener un mejor empleo y una mayor remuneración en el futuro.

Asimismo, las motivaciones para acceder a la educación superior dependen de múltiples factores que pueden agruparse en dos categorías. Por un lado, las cuestiones académicas, que hacen referencia a la variedad de oferta académica disponible para el interesado y las características de dicha oferta. Por otro lado, las cuestiones psicosociales, que se refieren a los factores que son derivados de la construcción individual y colectiva sobre lo que es beneficioso para el desarrollo del individuo, es decir, los idearios sobre educación superior como medio para lograr la movilidad social, conseguir mejores oportunidades laborales y la necesidad de contar con formación superior por presión social del grupo de referencia del individuo.

En Argentina, estudios previos muestran que los estudiantes eligen cursar estudios superiores para mejorar sus oportunidades de conseguir empleo, es decir, la razón instrumental prima en la decisión de ingreso (Kisilevsky, 2002; Veleda, 2002; Brito Cabrera y Ramos Velásquez, 2017). Además, influyen el nivel socio económico del estudiante, el nivel educativo de los padres y el rendimiento en la escuela media (Kisilevsky, 2002).

Sobre la base de las consideraciones teóricas y empíricas mencionadas, este trabajo analiza los aspectos que influyen en la decisión de acceder a la educación superior. Concretamente, la investigación gira en torno a la pregunta sobre los factores que influyeron en la decisión de ingresar y analiza como caso de estudio el acceso a la carrera de Tecnicatura Universitaria en Administración que se dicta en la Extensión Áulica San Martín de los Andes de la Facultad Regional del Neuquén de la Universidad Tecnológica Nacional (FRN-UTN).

La investigación contribuirá a profundizar el conocimiento sobre los determinantes de la decisión de acceder a estudios superiores, identificando los factores clave y su relevancia. En particular, contribuirá a la compresión del perfil de los estudiantes universitarios de San Martín de los Andes, así como sus motivaciones para acceder a estudios de educación superior y sus expectativas futuras respecto del egreso. En este sentido, el trabajo es novedoso ya que no se cuenta en la localidad con estudios previos similares sobre la temática planeada. Por lo tanto, el trabajo permite expandir el conocimiento sobre la demanda de educación en la localidad, la que presenta un importante crecimiento poblacional en los últimos años que hace prever un aumento de la demanda de educación en todos los niveles.

El trabajo se organiza de la siguiente manera. En el próximo apartado se mencionan los antecedentes teóricos de la temática planteada y los resultados de estudios empíricos previos. Luego se describen la metodología del trabajo y en particular el estudio llevado a cabo para profundizar el conocimiento sobre las motivaciones de los actuales estudiantes de una carrera de Tecnicatura Universitaria en Administración dictada en la localidad de San Martín de los Andes en la Extensión Áulica de la FRN-UTN. A continuación se presentan y analizan los resultados del trabajo empírico y finalmente las conclusiones.

\section{ANTECEDENTES}

En Argentina existen pocos estudios que tratan el tema de las motivaciones para el acceso a las instituciones de educación superior. La pregunta de qué motiva a una persona a optar por estudiar carece de una respuesta única.

Desde la teoría económica, la pregunta mencionada encuentra una respuesta en la teoría del capital humano (Morduchowicz, 2004) que explica la demanda de educación como resultado de los retornos que ésta otorga. Según esta teoría, la decisión de estudiar es una decisión de inversión en capital humano, es decir, una actividad que repercute en los ingresos 
monetarios futuros a través del incremento de los recursos incorporados en los individuos. Desde este enfoque, la educación (formal o no) permite al individuo incorporar capital humano, es decir, mejora sus capacidades y habilidades y esto le permite conseguir empleos mejor remunerados, estableciéndose así una relación causal y positiva entre el nivel educativo y los ingresos. Desde esta perspectiva, la demanda de educación superior depende de las expectativas sobre el mercado laboral, en particular, de obtener un mejor empleo y una mayor remuneración en el futuro.

Los estudios previos muestran que la educación es fundamental para garantizar la igualdad de oportunidades y la movilidad social ascendente. La evidencia empírica señala que el nivel educativo y el de ingresos de una persona están directamente relacionados (Pessino, 1996; Gasparini et al., 2001; Adrogué, 2006). Además, los retornos de la educación son positivos, es decir, a mayor nivel educativo mayores ingresos de la persona. (Psacharopoulos y Patrinos, 2002; Lema y Casellas, 2009).

El aprendizaje requiere una inversión, tanto por parte de la familia como del estudiante y por lo tanto, la demanda de educación depende de las condiciones socioeconómicas del estudiante y su familia. Por un lado, se encuentran los costos propios de los estudios, como los aranceles de las instituciones educativas. Por otro lado, están los costos de oportunidad, es decir, el uso del tiempo dedicado al estudio que deja de destinarse para realizar una actividad alternativa que podría generar ingresos para la persona. Aún con aranceles gratuitos, los estudios ocasionan gastos para la estudiante, tales como transporte, materiales de estudio, entre otros. Por otra parte, el "oficio de estudiante" requiere de una importante inversión de tiempo y esfuerzo. Por lo tanto, no bastan las oportunidades de inscripción e ingreso a una institución, sino que es preciso sostener la escolaridad para avanzar en la carrera y lograr la graduación.

Sin embargo, otros factores también influyen en la decisión y son parte de un marco explicativo del fenómeno de elección de un estudio de nivel superior que abarca factores económicos, sociales y psicológicos. En particular, las oportunidades de acceso al nivel superior contienen elementos atribuibles a las expectativas sociales, condicionantes académicos previos (en particular los resultados de la escuela media) y el nivel socioeconómico del estudiante y su familia. Influyen en la decisión las expectativas de los padres, el apoyo y el estímulo de la familia, los compañeros y los docentes del colegio secundario; el desarrollo de claras aspiraciones ocupacionales y educacionales; las experiencias en la escuela secundaria; los recursos académicos que brinda el nivel de enseñanza media; el acceso a información sobre lo que ofrecen las universidades; la disponibilidad de ayuda financiera para solventar los gastos vinculados con los estudios; la preparación para los exámenes de ingreso; las experiencias de otros en los estudios superiores; y las responsabilidades familiares (Adelman, 1999; Cabrera y La Nasa, 2001).

En una recopilación de diferentes trabajos realizados sobre los perfiles y motivaciones de los estudiantes de escuelas medias para continuar estudios superiores Kisilevsky (2002) encuentra que el nivel socio económico del estudiante así como el nivel educativo de los padres y el rendimiento en la escuela media son factores relevantes que explican la decisión de ingreso a la universidad. Asimismo, destaca que los estudiantes ingresan a la universidad con un déficit académico del nivel medio y que tienen escasa información sobre la universidad previamente a ingresar a ella. Del mismo modo, las instituciones de educación superior en general desconocen el perfil de sus estudiantes, lo que dificulta el tránsito por los estudios superiores sobre todo en el primer año de cursado.

Por su parte, Veleda (2002) analiza las estrategias que intervienen en la decisión de 
ingresar a la universidad y elegir una casa de estudios distinguiendo las motivaciones de los estudiantes según su nivel socioeconómico. En su trabajo, destaca que, ante la pregunta de por qué decidieron continuar los estudios, el grupo de jóvenes de sectores medios bajos sostiene unánimemente que se debe a que actualmente el título secundario no es suficiente para obtener un trabajo, observándose así que es la razón instrumental la que prima en la argumentación de estos estudiantes, lo cual no implica la ausencia de otras razones tales como el interés por una disciplina en particular, aunque éstas son claramente secundarias en el orden de prioridades. Siguiendo con la misma investigación, la autora destaca que, si bien los jóvenes de sectores medios-altos también mencionan la necesidad de valerse de un título universitario o terciario para hacer frente al mercado laboral, las razones vocacionales o de realización personal están mucho más presentes. Finalmente, concluye que la diferencia entre las motivaciones que llevan a los jóvenes de sectores medios altos y bajos a continuar sus estudios está íntimamente ligada a las posibilidades que tienen dichos grupos de comenzar sus estudios superiores.

Brito Cabrera y Ramos Velásquez (2017) indagan acerca del perfil de los ingresantes en una universidad argentina. En su estudio encuentran que entre los motivos principales para estudiar una carrera universitaria, los encuestados respondieron que lo hacían para tener trabajo, para mejorar su nivel socioeconómico, y para obtener reconocimiento social y familiar, siendo estos los motivos más mencionados.

De la revisión de estudios previos se pueden extraer algunas conclusiones relevantes. Por un lado, cabe destacar que las motivaciones respecto del ingreso a la educación superior son altas, sea por la presión familiar o por la expectativa de lograr mejores oportunidades laborales en el futuro, en comparación con la baja tasa de graduación por habitante, lo que determina un problema serio de abandono en el sistema de educación superior. Estas aspiraciones por cursar estudios superiores no siempre se materializan por razones individuales y también institucionales. En general, cuanto mayor es el nivel socioeconómico del estudiante, mejores sus calificaciones en la etapa previa de estudios, más alto es el nivel educativo de los padres mayores son las expectativas de continuar estudios de nivel superior. Asimismo, el traslado de ciudad para estudiar es también un factor relevante, dado que los costos directos de la educación son considerablemente más elevados si el estudiante realiza estudios superiores fuera de su ciudad de origen. Esto hace que la distribución geográfica de la oferta académica pueda ejercer un impacto en las orientaciones ya que no todos los estudiantes pueden afrontar estos costos. Así, los datos socioeconómicos tienen un peso considerable en la definición de los perfiles.

\section{METODOLOGÍA}

En este apartado se describe la metodología de trabajo y las fuentes de información utilizadas.

El trabajo se enmarca en el tipo de proyectos de investigación aplicada. Para cumplir con los objetivos se realizó en primer lugar una revisión bibliográfica sobre la temática planteada con el objetivo de estudiar las perspectivas teóricas que dan sustento al tema de investigación propuesto. En particular se tuvieron en cuenta aquellos estudios que analizan la educación superior en contextos de gratuidad del arancel y acceso sin restricciones a las instituciones universitarias. En segundo lugar, se realizó una parte empírica para profundizar el conocimiento sobre las motivaciones de los actuales estudiantes de una carrera dictada en la localidad de San Martín de los Andes: la Tecnicatura Universitaria en Administración de la Extensión Áulica San Martín de los Andes de la FRN-UTN. 
La Extensión Áulica San Martín de los Andes de la FRN-UTN dicta desde el año 2018 la carrera de Tecnicatura Universitaria en Administración. Se trata de una carrera universitaria de 2 años de duración, con cursado presencial, asistencia obligatoria y no arancelada. Se cursa de lunes a viernes en la sede de la Extensión Áulica, ubicada en dependencias de la Secretaría de Educación de la Municipalidad local, en el centro de la ciudad. La carrera comenzó a dictarse en el año 2018 y contó con unos 80 estudiantes inscriptos que comenzaron a cursar.

Mediante la aplicación de una encuesta se indagó sobre los factores que influyeron en estos estudiantes para comenzar a cursar estudios superiores y en particular, la carrera mencionada, única en su oferta en la localidad. La encuesta fue abierta a todos los estudiantes que desearon participar y se aplicó entre los meses de octubre y noviembre de 2018. Consiste en un cuestionario con preguntas con opciones cerradas de respuesta y otras preguntas de respuesta abierta. Se indagó sobre cuestiones socioeconómicas de los estudiantes, aspectos vinculados con sus motivaciones para elegir la carrera y expectativas sobre su futuro laboral post egreso.

En particular, la encuesta tiene dos apartados. En un primer apartado se indaga sobre el contexto socioeconómico y demográfico del estudiante y su familia. Se incluyen preguntas sobre su edad, lugar de residencia, nivel máximo de formación académica de los padres y preguntas vinculadas con su situación laboral (si trabaja, si busca trabajo, modalidad laboral, entre otras). En el segundo apartado se indaga sobre el contexto formativo profesional del estudiante. Se preguntó sobre su nivel de formación, los motivos para inscribirse en la carrera de Tecnicatura Universitaria en Administración de la Extensión Áulica de la FRNUTN, los factores que influyeron en la decisión, las expectativas sobre el impacto de la formación superior en su futuro laboral y las perspectivas de continuar estudios superiores una vez finalizada la carrera elegida. Se preguntó en qué medida la decisión de cursar los estudios universitarios estuvo influida por cuestiones como la duración de la carrera, la disponibilidad del cursado presencial en la localidad y la gratuidad del arancel, entre otras. Las preguntas de los apartados mencionados son con opciones cerradas de respuesta, dando siempre una opción de "Otros" para que el encuestado pueda volcar su propia respuesta. Finalmente, se incluyó un espacio abierto donde los encuestados podrán dejar comentarios y reflexiones vinculados con las preguntas formuladas previamente.

Como se mencionó antes, se invitó a todos los estudiantes de la carrera a participar de la investigación y se les facilitó el formulario de encuesta en formato impreso y por correo electrónico. Las respuestas fueron procesadas manualmente y los resultados fueron volcados en un software de hoja de cálculo para su análisis. Se procesaron un total de 24 encuestas. A continuación se presentan los resultados.

\section{RESULTADOS Y DISCUSIÓN}

En este apartado se presentan y analizan los resultados del estudio empírico realizado a la población estudiantil de la Extensión Áulica San Martín de los Andes de la FRN-UTN.

La encuesta fue respondida por 24 estudiantes, cantidad que representa el $40 \%$ del total que cursaba en la carrera en el momento de la aplicación del cuestionario (noviembre de 2018). Los números y porcentajes que se presentan en esta sección corresponden a los estudiantes que respondieron la encuesta, la que, como se mencionó, fue abierta a toda la población estudiantil de la carrera. A continuación se presentan los principales resultados. 


\section{CONTEXTO SOCIOECONÓMICO Y DEMOGRÁFICO}

En el primer apartado de la encuesta se realizaron preguntas vinculadas con el perfil socioeconómico y demográfico de los estudiantes. El objetivo de esta sección es describir al estudiantado de la carrera en función de algunas características vinculadas con su situación familiar y laboral.

Los estudiantes que respondieron la encuesta viven en su totalidad en San Martín de los Andes, ciudad de donde la mayoría (63\%) es oriundo. El 56\% tienen más de 25 años y a su vez 1 de cada 5 tiene 36 años o más.

Todos los estudiantes que respondieron la encuesta viven en un entorno familiar. El 54\% convive con sus padres, el 33\% con su pareja e hijos y el resto con sus hijos.

En relación con la situación laboral (Gráfico 1), la mayoría de los estudiantes trabaja, aunque sólo muy pocos lo hacen a tiempo completo. Sólo 2 de los 18 estudiantes que trabajan están buscando otro empleo: uno de ellos trabaja en forma esporádica y el otro lo hace en forma regular, menos de 20 horas semanales. Entre los estudiantes que no trabajan, el 80\% está buscando empleo.

En el grupo etario de los estudiantes más jóvenes (18 a 25 años), la mitad no trabaja aunque todos están buscando empleo. Por el contrario, los estudiantes con más edad (36 años en adelante) están en su totalidad empleados aunque ninguno a tiempo completo (60\% trabaja a tiempo parcial y el resto trabaja menos de 20 horas semanales). Asimismo, ninguno manifestó estar buscando empleo.

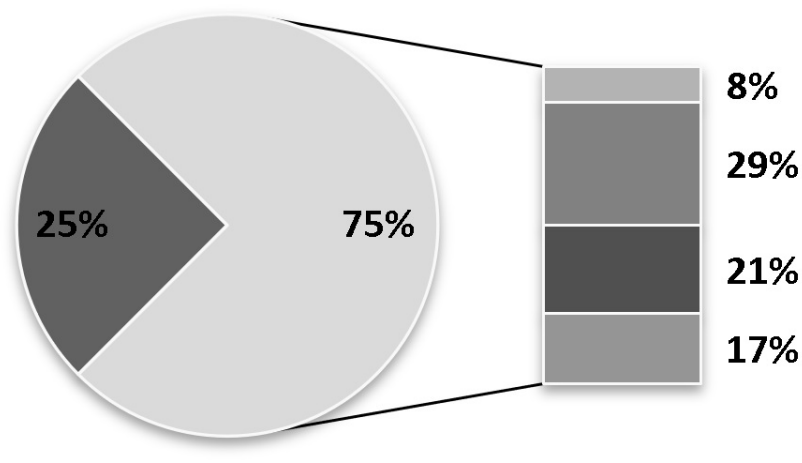

No trabaja

$8 \%$

$29 \%$

- Trabaja 40 horas semanales o más

Trabaja entre 20 y 40 horas semanales

- Trabaja menos de 20 horas semanales

Gráfico 1. Situación laboral de los estudiantes encuestados

En relación con la modalidad laboral (Gráfico 2), la mayoría de los que trabaja lo hace en relación de dependencia (en el sector público o privado).

Respecto a la duración en el trabajo, la mitad de los estudiantes que trabaja cuenta con una antigüedad inferior al año en su actual empleo. Los que tienen más antigüedad en su trabajo (3 años o más) representan el 33\% de los que trabajan y son todos estudiantes que tienen 26 años de edad o más. 


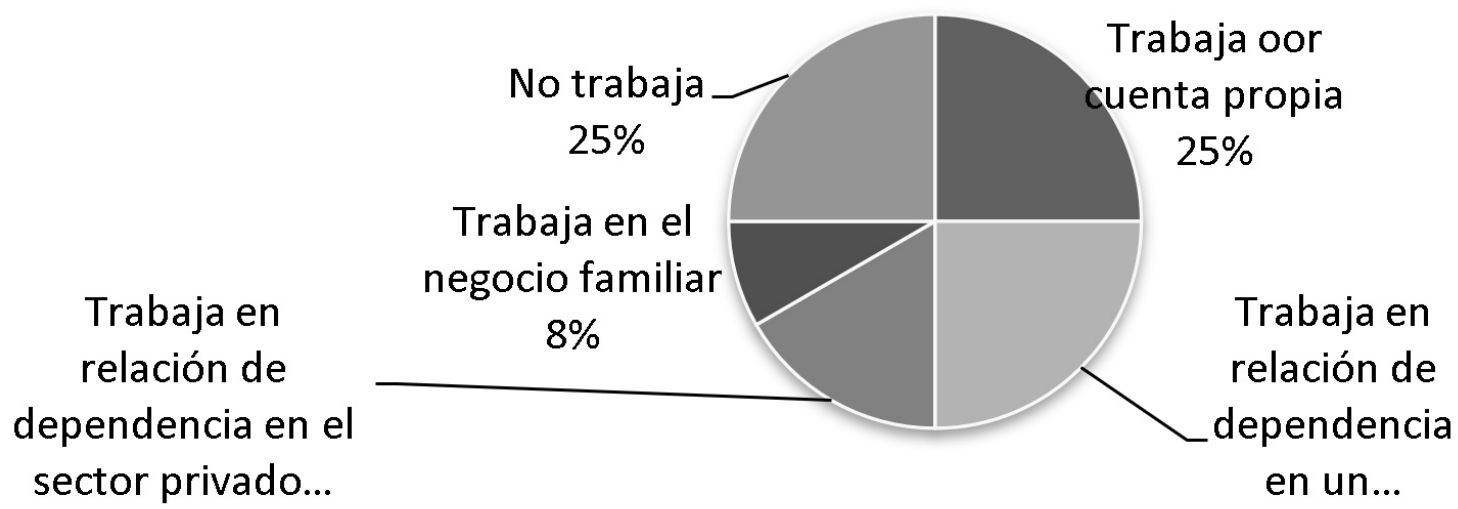

Gráfico 2. Modalidad laboral de los estudiantes encuestados

En relación con el entorno familiar, se indagó acerca del perfil educativo de los padres de los estudiantes. Las respuestas fueron heterogéneas pero se destaca que sólo uno de los estudiantes que respondió la encuesta proviene de un hogar donde ambos progenitores tienen formación universitaria completa; asimismo, 4 estudiantes (17\% del total de encuestados) provienen de un hogar donde al menos uno de los progenitores tiene formación universitaria (completa o no). La categoría con mayor nivel de frecuencia de resultados es la de primario completo, nivel de formación máximo alcanzado por el $29 \%$ de los padres y madres de los estudiantes que participaron de la encuesta.

En síntesis, los estudiantes de la carrera viven en la localidad de San Martín de los Andes, en un entorno familiar y son, en su mayoría, primera generación de estudiantes universitarios. Asimismo, la mayoría son estudiantes de tiempo parcial puesto que, comparten la actividad académica con la actividad laboral y la vida familiar.

\section{CONTEXTO FORMATIVO}

En el segundo apartado de la encuesta se realizaron preguntas relacionadas con el perfil formativo de los estudiantes. Concretamente se indagó sobre los antecedentes en educación superior de los estudiantes y las experiencias pasadas en dicho ámbito.

El 75\% de los estudiantes que respondieron la encuesta tenía alguna experiencia en el ámbito de la educación superior antes de inscribirse en la Tecnicatura Universitaria en Administración de la FRN-UTN, ya que cursaron alguna carrera universitaria previa (Gráfico 3). El 25\% restante cuenta con el secundario completo como nivel máximo de formación académica alcanzado y todos ellos hicieron algún curso no universitario antes de inscribirse en la Tecnicatura. 


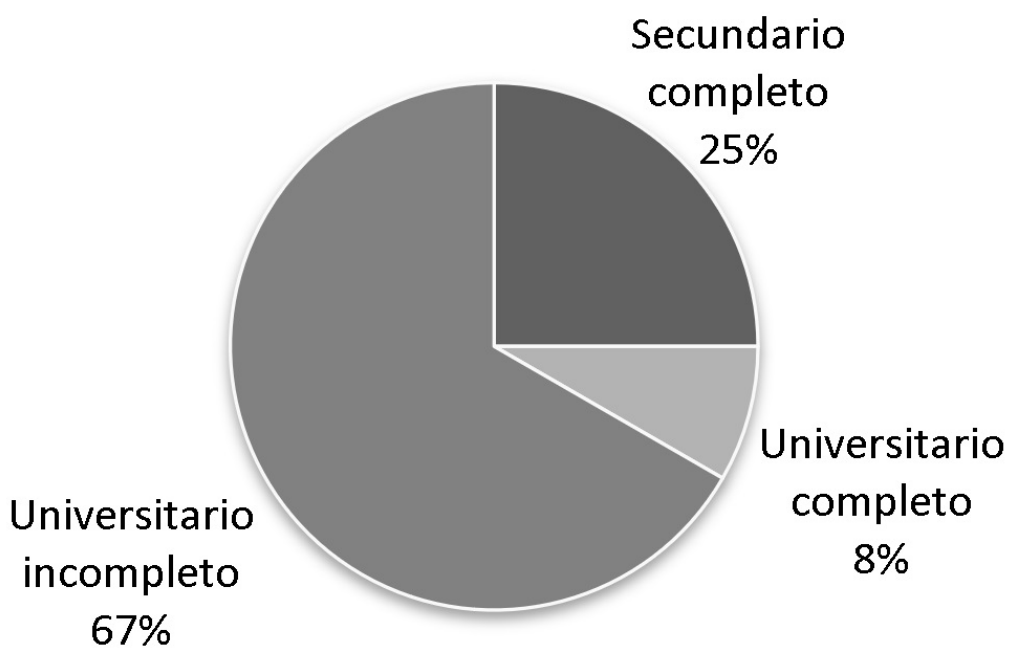

Gráfico 3. Nivel educativo máximo de los encuestados al ingresar a la carrera

Sólo 2 estudiantes que respondieron la encuesta (8\%) cuentan con un título universitario antes de inscribirse en la Tecnicatura. Ambos cursaron y aprobaron propuestas académicas en modalidad presencial y con asistencia obligatoria.

El 67\% del total de estudiantes que respondieron la encuesta abandonó los estudios previos. Entre ellos, la mitad cursaba carreras presenciales con asistencia obligatoria y la otra mitad cursaba carreras en la modalidad a distancia. Las causas por las cuales abandonaron los estudios son variadas. Las principales son los problemas para cursar por superposición horaria con otras actividades (21\%) y las dificultades para seguir los estudios con la modalidad a distancia (21\%). Otros problemas mencionados fueron las dificultades económicas (16\%) la falta de interés por los contenidos (8\%).

En síntesis, la mayoría de los estudiantes cuenta con antecedentes en el sistema de educación superior y comenzaron a cursar la Tecnicatura Universitaria en Administración luego de haber estudiado otra carrera. Sin embargo, la mayoría no logró completar las instancias previas de formación. La cuestión vocacional no aparece como un motivo preponderante para justificar el abandono de los estudios previos. Las razones de los fracasos previos tienen que ver con las dificultades para congeniar el estudio con otras actividades (laborales o familiares), situación relevante ya que en su mayoría se trata de estudiantes de tiempo parcial, puesto que la mayoría trabaja, y en muchos casos tienen hijos. También es importante como causa de abandono la dificultad para cursar estudios en la modalidad a distancia. Estas dificultades tienen que ver con factores personales, como la falta de motivación y predisposición al aprendizaje guiado y factores vinculados con la experiencia universitaria, como la necesidad de una adecuada organización o método de estudio (Vásquez Martínez, C y otro, 2007). También son relevantes factores externos al estudiante, como la falta de apoyo familiar, conflicto de horarios o no disponer de tiempo suficiente y las dificultades económicas. Finalmente, también influyen factores propios de la naturaleza de la propuesta educativa, como el insuficiente apoyo docente o tecnológico o falta de soporte organizacional 
(Escanés, G y otros, 2014).

\section{MOTIVACIONES PARA EL INGRESO}

Al ser consultados sobre las razones para inscribirse a cursar la carrera, los estudiantes que respondieron la encuesta valoraron la formación académica y la perspectiva laboral futura (Gráfico 4). El 83\% de los estudiantes indicaron que se inscribieron a la carrera porque querían mejorar su formación académica mientras que el 79\% indicaron que lo hicieron para acceder a mejores oportunidades laborales en el futuro. Además, el 54\% se inscribió porque le gusta el área temática de la administración.

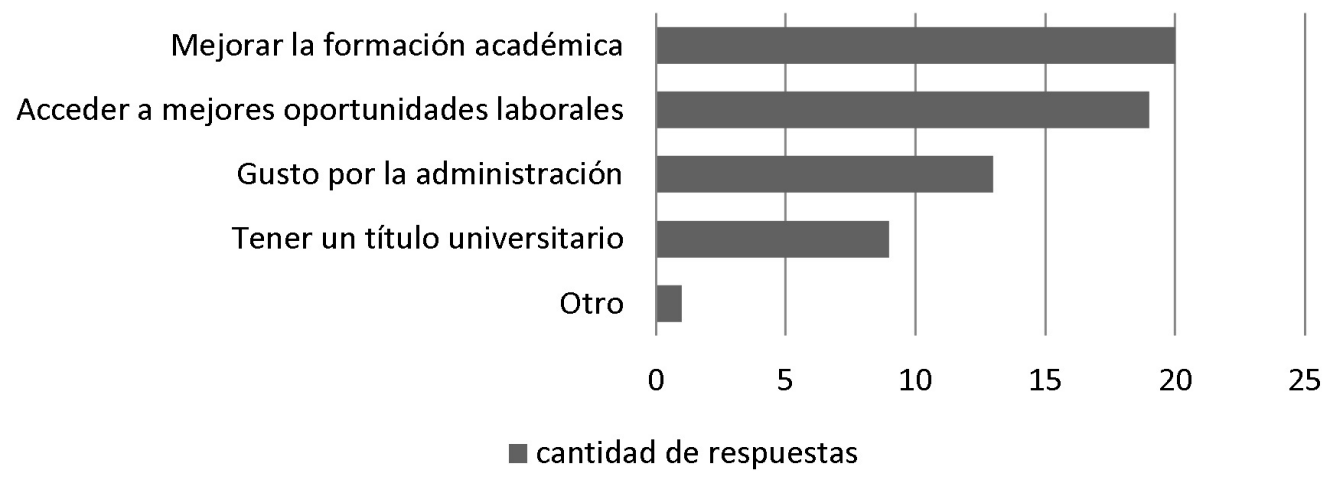

Gráfico 4. Razones principales de los encuestados para inscribirse a cursar la carrera

La necesidad y el deseo de contar con un título de educación superior aparecen como aspectos muy valorados por los estudiantes encuestados a la hora de decidir inscribirse a la carrera. El 63\% de los encuestados manifestó que la necesidad de contar con un título, por ejemplo por motivos laborales, fue un factor muy importante a la hora de decidir inscribirse en la carrera.

Por su parte, el 79\% manifestó el deseo de contar un título como un aspecto muy importante o algo importante. De ellos, el 74\% ya tenía una experiencia previa en el ámbito universitario, aunque solo uno de los estudiantes logró completar los estudios superiores previos.

En la encuesta también se indagó sobre la influencia de algunas características de la carrera en la decisión de los estudiantes de inscribirse a cursar (Gráfico 5). En particular, se analizó la duración de la carrera, la gratuidad del arancel, la modalidad de cursado, la localización del cursado, el área curricular y la reputación de la UTN. La influencia se evaluó con cuatro opciones: impacto determinante (sin esa característica no se hubiera inscripto a cursar), impacto positivo, poco impacto e impacto nulo. Además, se indagó sobre la probabilidad de inscribirse a cursar si se presentaban ciertas características diferentes a las que tenía la oferta académica considerada, como son el cursado en otra localidad, en modalidad a distancia, arancelado, entre otras. 


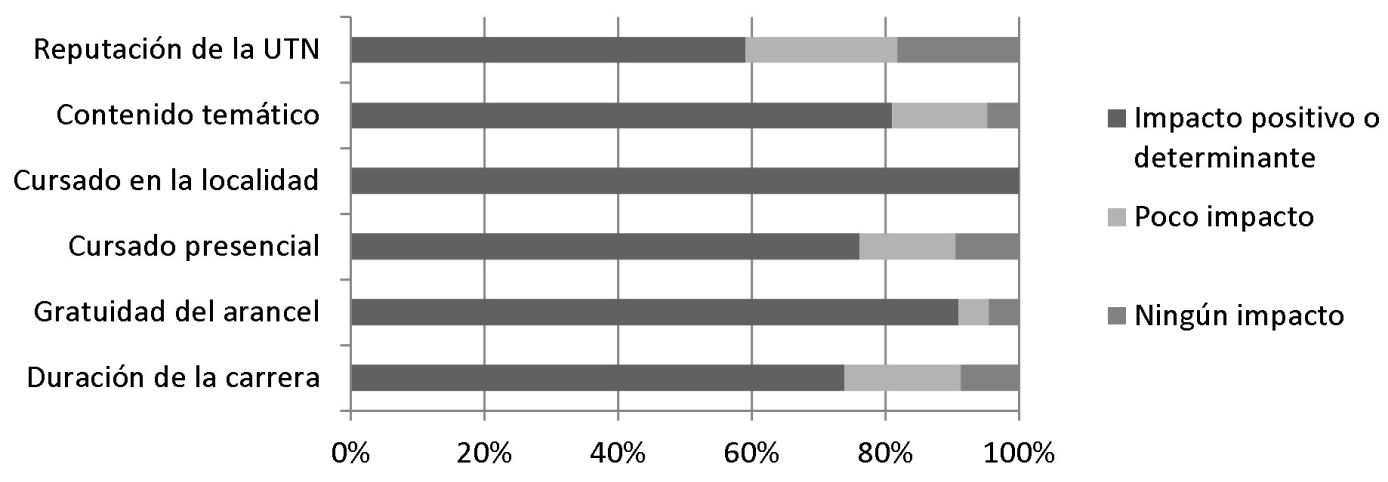

Gráfico 5. Impacto de características de la carrera en la decisión estudiar

Todos los encuestados consideraron el cursado en la localidad como un factor con impacto positivo, mientras que el 75\% la consideró una condición determinante para decidir la inscripción a la carrera. Cabe destacar que esta característica es la única de las evaluadas que no tuvo respuestas en las opciones "poco impacto" o "impacto nulo", poniendo de manifiesto la importancia del cursado en la localidad para decidirse a estudiar. Asimismo, el 65\% consideró improbable haberse inscripto a cursar la carrera si se dictaba en otra localidad mientras que el 30\% lo consideró poco probable.

La relevancia del dictado en la localidad queda de manifiesto en estas tres opiniones recogidas en las encuestas:

"existe un gran interés de los alumnos que esta carrera continúe siendo dictada en la localidad" (estudiante entre 36 y 45 años que trabaja entre 20 y 40 horas semanales)

"Ha sido una gran oportunidad que nos han dado al traer esta carrera a aquellos que por diferentes motivos no pudimos estudiar. Espero que en algún momento se vuelva abrir esta carrera para darle oportunidad a otras personas.” (estudiante entre 26 y 35 años que trabaja en forma esporádica)

"Considero que la presencia de la extensión áulica de la UTN en la localidad nos permite acceder a nuevas carreras y muy requeridas. Quienes estamos acostumbrados al "pueblo chico" tenemos serias dificultades para irnos a estudiar a otros lugares porque sufrimos la adaptación." (estudiante entre 26 y 35 años que trabaja entre 20 y 40 horas semanales)

El cursado de estudios superiores en la misma localidad de residencia del estudiante es relevante por diferentes motivos. Por un lado, supone un ahorro importante de recursos financieros dado que evita los costos asociados con el traslado para estudiar. Esto hace que la distribución geográfica de la oferta académica de nivel superior pueda ejercer un impacto 
en las orientaciones vocacionales ya que no todos los estudiantes pueden afrontar estos costos de traslado, que pueden ser considerables. Por otro lado, el cursado en la localidad permite una adaptación menos compleja del estudiante a sus nuevas responsabilidades. Esto es porque se evitan las cuestiones emocionales y psicológicas vinculadas con el desarraigo al permitirle al estudiante seguir conviviendo con su círculo familiar y social y así adaptarse mejor a sus nuevas actividades.

Adicionalmente, más del $40 \%$ de los estudiantes encuestados consideraron determinante el cursado presencial, mientras que el 33\% consideró esta característica con un impacto positivo. La mitad de los encuestados evaluó como improbable haberse inscripto si la oferta académica se dictaba en la modalidad a distancia mientras que el 38\% lo consideró poco probable.

Otra condición de la carrera con un alto porcentaje de respuestas con impacto determinante o positivo fue la gratuidad del arancel; 9 de cada 10 estudiantes consideraron esta condición en las categorías mencionadas mientras que 4 de cada 10 la consideró determinante. El 86\% evaluó como poco o nada probable inscribirse a cursar si la carrera fuera arancelada.

La importancia de la gratuidad queda de manifiesto en esta opinión recogida en una de las encuestas:

\footnotetext{
"Lamentablemente (en San Martín de los Andes) la oferta académica gratuita es la misma desde hace muchos años y quienes desean estudiar algo diferente tienen que pagar y es muy costoso." (estudiante entre 26 y 35 años que trabaja entre 20 y 40 horas semanales)
}

Sólo el 21\% de los estudiantes consideró que los contenidos temáticos de la carrera (administración) fueron un factor determinante a la hora de inscribirse. Sin embargo, el 50\% lo consideró como un aspecto que tuvo un impacto positivo.

Respecto a la universidad donde se dicta la carrera, el 59\% consideró la reputación de la UTN con un aspecto con impacto positivo o determinante a la hora de inscribirse. El 82\% consideró probable haberse inscripto si la carrera era ofrecida por otra universidad.

Finalmente, se indagó acerca de las expectativas sobre la carrera, en particular, cómo impactaría en el futuro laboral contar con el título de Técnico Universitario en Administración. Los resultados arrojan que el 96\% de los estudiantes consideró que contar con el título universitario les permitirá mejorar su situación laboral (Gráfico 6). En particular, el 42\% consideró que le permitirá conseguir trabajo mientras que el 63\% expresa que el título le permitirá conseguir un trabajo mejor remunerado. 


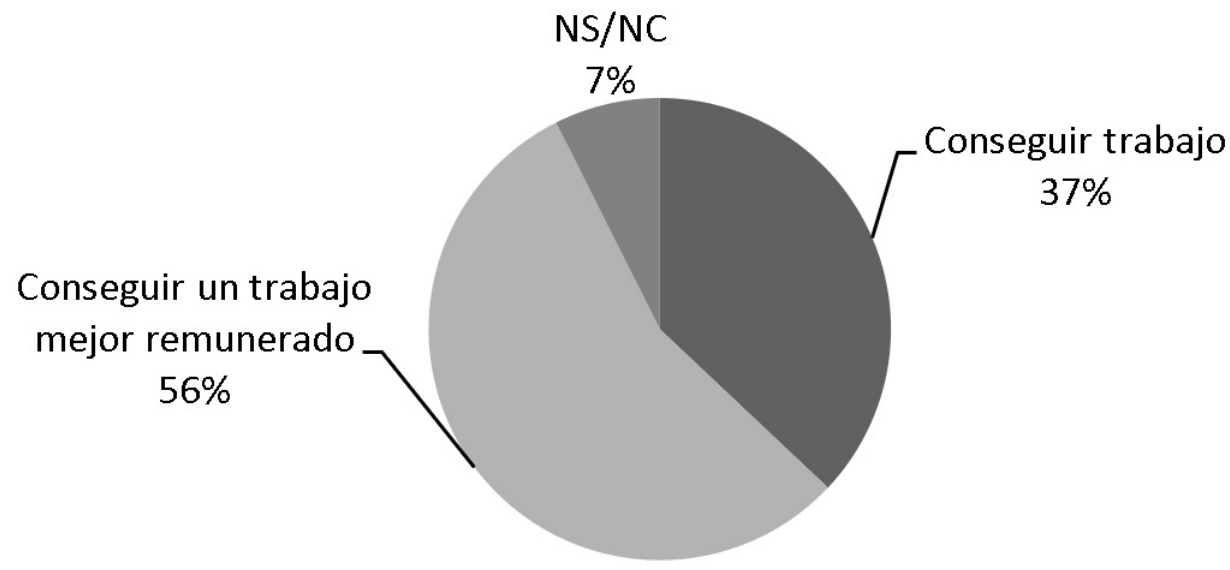

Gráfico 6. Expectativas sobre la formación universitaria en Técnico Universitario en Administración.

La valoración de la educación superior como mecanismo de movilidad social puede verse reflejada en este comentario recogido en una de las encuestas:

"Hoy puedo afortunadamente estudiar algo que me gusta y que estoy segura me permitirá crecer laboralmente" (estudiante entre

26 y 35 años que trabaja entre 20 y 40 horas semanales)

Asimismo, 3 de cada 4 estudiantes encuestados estima probable continuar estudios superiores una vez que finalizada la carrera de Técnico Universitario en Administración. De ellos, el 44\% tiene certeza que continuará estudiando.

Estos resultados refuerzan la relevancia de las cuestiones psicosociales vinculadas con la educación superior, en particular, el ideario de la educación superior como medio para lograr la movilidad social y conseguir mejores oportunidades laborales.

\section{CONCLUSIONES}

El aumento de la demanda es uno de los factores más relevantes del sistema educativo Sobre la base de las consideraciones teóricas y empíricas mencionadas, este trabajo analiza los aspectos que intervienen en la decisión de acceder a la educación superior. Concretamente, la investigación gira en torno a la pregunta sobre los factores que influyeron en la decisión de ingresar y analiza como caso de estudio el acceso a la carrera de Tecnicatura Universitaria en Administración que se dicta en la Extensión Áulica San Martín de los Andes de la Facultad Regional del Neuquén de la Universidad Tecnológica Nacional (FRN-UTN).

El estudio empírico permitió identificar los factores que influyeron en los estudiantes de San Martín de los Andes para comenzar a cursar estudios superiores en la FRN-UTN y en particular, la carrera de Tecnicatura Universitaria en Administración.

De la investigación realizada se puede concluir que las motivaciones para ingresar a la 
universidad son altas, en particular por la expectativa de lograr mejores oportunidades laborales. Las principales motivaciones para estudiar corresponden a factores instrumentales vinculados con el deseo y la necesidad de contar con un título de educación superior y a cuestiones psicosociales, en particular, el ideario de la educación superior como medio para lograr la movilidad social y conseguir mejores oportunidades laborales. Es interesante observar que el deseo de contar con un título de educación superior tiene una valoración más importante en cuanto a la motivación para estudiar que la vocación por el área disciplinar propia de la oferta académica en cuestión, lo que muestra nuevamente la relevancia del factor instrumental.

De la indagación sobre las características de la carrera que resultaron atractivas para motivar el ingreso, surge claramente la relevancia del cursado local, presencial y gratuito. Estas características se explican principalmente por las particularidades del alumnado de la carrera: la mayoría son estudiantes de tiempo parcial y que viven en entornos familiares y por lo tanto comparten la actividad académica con la actividad laboral y la vida familiar. La dificultad para el traslado de ciudad para estudiar hace que la distribución geográfica de la oferta académica pueda ejercer un impacto en las orientaciones ya que no todos los estudiantes pueden afrontar estos costos. Así, los datos socioeconómicos tienen un peso considerable en la definición de los perfiles.

La oferta académica de la FRN-UTN se posiciona como una opción de formación superior que permite captar un estudiantado que difícilmente, por sus características (estudiantes de tiempo parcial que viven en un entorno familiar y son en su mayoría primera generación de estudiantes universitarios), hubiera demandado educación superior si no tuviera esta oportunidad de formación. En este sentido, los resultados refuerzan la idea de que la existencia de nuevas localizaciones de oferta de educación superior puede constituir un incentivo para la expansión de la demanda. Un aspecto a analizar es en qué medida la sola ampliación de la oferta local de educación superior contribuye a la inclusión social al facilitar no sólo que las personas accedan a los estudios de nivel superior sino también que gocen de una formación académica y profesional apropiada que les permita insertarse exitosamente en el mercado laboral.

Para finalizar, se destaca que la investigación contribuye a profundizar el conocimiento sobre los determinantes de la decisión de acceder a estudios superiores, identificando los factores clave y su relevancia. En particular, aporta a la compresión del perfil de los estudiantes universitarios de San Martín de los Andes, así como sus motivaciones para acceder a estudios de educación superior y sus expectativas futuras respecto del egreso. En este sentido, el trabajo es novedoso ya que no se cuenta en la localidad con estudios previos similares sobre la temática planeada. Por lo tanto, el trabajo permite expandir el conocimiento sobre la demanda de educación en la localidad, la que presenta un importante crecimiento poblacional en los últimos años que hace prever un aumento de la demanda de educación en todos los niveles.

Los resultados afianzan el ideario de la educación superior como un elemento necesario para acceder a mejores oportunidades laborales y destacan el valor intrínseco de la formación académica materializada en un título. También ponen de manifiesto la relevancia de contar con ofertas educativas locales en contextos donde prima una baja movilidad estudiantil por motivos económicos, familiares o idiosincráticos.

Los resultados son relevantes para las autoridades de la Universidad Tecnológica Nacional y en particular la Facultad Regional del Neuquén de la que depende la Extensión Áulica San Martín de los Andes por cuanto les permite contar con información relevante sobre los 
factores que influyen en la decisión de acceso a la universidad. Esta información puede ser utilizada luego como un insumo para orientar la oferta educativa de la Extensión Áulica de acuerdo con las demandas locales. Adicionalmente, los resultados son de utilidad para los hacedores de política educativa en el ámbito municipal y regional ya que podrán considerar los resultados obtenidos para diseñar e implementar políticas educativas que potencien las posibilidades de acceso a la educación superior. 


\section{REFERENCIAS}

Adelman, C (1999) "Answers in the tool box: Academic intensity, attendance patterns, and bachelor's degree attainment”. Document \# PLLI 19998021. Washington DC: U.S. Department of Education, Office of Educational Research and Improvement, EEUU. Disponible en https://files.eric.ed.gov/fulltext/ED431363.pdf

Adrogué, C (2006) “Desempleo y retornos a la educación en la Argentina (1974-2002)”. Anales de la Asociación Argentina de Economía Politica. Disponible en https://aaep.org.ar/ anales/works06/AdrogueCecilia.pdf.

Brito Cabrera, C y Ramos Velásquez, M (2017) "Estudio sobre las características del perfil del ingresante de la Universidad del Chubut" VI Congreso Nacional e Internacional de Estudios Comparados en Educación. Buenos Aires, septiembre de 2017. Disponible en http://www.saece.com.ar/docs/congreso6/trab017.pdf

Cabrera, A y La Nasa, S (2001) "On the path to college: Three critical tasks facing America's disadvantaged". Research in Higher Education, 42(2). Disponible en https://www. researchgate.net/publication/225876350_On_the_Path_to_College_Three_Critical_Tasks_ Facing_America's_Disadvantaged.

Escanés, G; Herrero, V; Merlino, A y Ayllón, S (2014) "Deserción en educación a distancia: factores asociados a la elección de modalidad como desencadenantes del abandono universitario". Virtualidad, Educación y Ciencia, 9 (5). Disponible en file://C:/Users/Marita/ Downloads/9549-Texto\%20del\%20art\%C3\%ADculo-25589-1-10-20141205.pdf

García de Fanelli, A (2015) "Inclusión social en la Educación Superior Argentina: indicadores y políticas en torno al acceso y a la graduación.” Páginas De Educación, 7(2). Disponible en https://revistas.ucu.edu.uy/index.php/paginasdeeducacion/article/view/510.

Gasparini, L.; Marchionni, M. y Sosa Escudero, W. (2001) "Distribución del Ingreso en la Argentina. Perspectivas y Efectos sobre el Bienestar”. Premio Fulvio Salvador Pagani 2001, Fundación Arcor, Buenos Aires, Argentina. ISBN: 987-9449-89-4. 2001.

IIEP (2017) "Informe de Seguimiento de la educación en el mundo". UNESCO. Disponible en https://unesdoc.unesco.org/ark:/48223/pf0000261016_spa

Kisilevsky, M (2002) "Condiciones sociales y pedagógicas de ingreso a la educación superior en la Argentina” en Kisilevsky, M y Veleda C "Dos estudios sobre el acceso a la educación superior en la Argentina” IIPE - UNESCO. Disponible en https://unesdoc. unesco.org/ark:/48223/pf0000129506?posInSet=122\&queryId=a895b224-be77-45b3-91daf91753d3130c

Lema, D. y Casellas, K. (2009) “Retornos a la educación en zonas rurales”. Revista Argentina de Economía Agraria. Volumen XI Número 1. Primavera 2009. ISSN 0327-3318. Disponible en https://inta.gob.ar/sites/default/files/script-tmp-retornos_educacion_zonas_rurales_ lema_casellas.pdf

Ministerio de Educación (2019) “Estadísticas Universitarias” Disponibles en http:// estadisticasuniversitarias.me.gov.ar/\#/home

Morduchowicz, A (2004) “Discusiones de economía de la educación” Editorial Losada. Buenos Aires, Argentina. 
Psacharopoulos, G. y Patrino, H. (2002) "Returns to Investment in Education: A further update”. World Bank Policy Research Working Paper 2881. Disponible en https:// openknowledge.worldbank.org/handle/10986/19231

Pessino, C (1996) "Returns to Education in Greater Buenos Aires 1986-1993: From Hyperinflation to Stabilization”. Cuadernos de Economía 33(99): 205-226. CEMA. Disponible en https://ucema.edu.ar/publicaciones/download/documentos/104.pdf.

Vásquez Martínez, C y Rodríguez Pérez, M.C. (2007) "La deserción estudiantil en educación superior a distancia: perspectiva teórica y factores de incidencia". Revista Latinoamericana de Estudios Educativos, 37 (3-4), pp. 107-122. Disponible en https://www.redalyc.org/ pdf/270/27011410005.pdf

Veleda, C (2002) "Estrategias individuales y familiares en la elección de las instituciones de educación superior" en Kisilevsky, M y Veleda C "Dos estudios sobre el acceso a la educación superior en la Argentina” IIPE - UNESCO. Disponible en https://unesdoc. unesco.org/ark:/48223/pf0000129506?posInSet=122\&queryId=a895b224-be77-45b3-91daf91753d3130c 